\title{
Direct comparison of rapid deployment versus sutureless aortic valve replacement: a meta-analysis
}

\author{
Suk Ho Sohn ${ }^{1} \wedge$, Yoonjin Kang ${ }^{1} \wedge$, Ji Seong Kim ${ }^{1} \wedge$, Jae Woong Choi ${ }^{1} \wedge$, Myoung-Jin Jang ${ }^{2 \#}$, \\ Ho Young Hwang ${ }^{1 \# \wedge}$
}

${ }^{1}$ Department of Thoracic and Cardiovascular Surgery, Seoul National University Hospital, Seoul National University College of Medicine, Seoul, Korea; ${ }^{2}$ Medical Research Collaborating Center, Seoul National University Hospital, Seoul National University College of Medicine, Seoul, Korea Contributions: (I) Conception and design: SH Sohn, HY Hwang; (II) Administrative support: HY Hwang; (III) Provision of study materials or patients: HY Hwang, Y Kang, JS Kim, JW Choi; (IV) Collection and assembly of data: SH Sohn, Y Kang, JS Kim, JW Choi; (V) Data analysis and interpretation: SH Sohn, MJ Jang; (VI) Manuscript writing: All authors; (VII) Final approval of manuscript: All authors.

"These authors contributed equally to this work.

Correspondence to: Ho Young Hwang, MD, PhD. Department of Thoracic and Cardiovascular Surgery, Seoul National University Hospital, Seoul National University College of Medicine, 101 Daehak-ro, Jongno-gu, Seoul 03080, Korea. Email: scalpel@hanmail.net; Myoung-Jin Jang, PhD. Medical Research Collaborating Center, Seoul National University Hospital, Seoul National University College of Medicine, 101 Daehak-ro, Jongno-gu, Seoul 03080, Korea. Email: mjjang2014@naver.com.

Background: This meta-analysis was conducted to compare the procedural and early outcomes of aortic valve replacement (AVR) using rapid deployment valve (RD group) versus sutureless valve (SU group).

Methods: A literature search of 5 online databases was conducted. The primary outcomes were mean transvalvular pressure gradient (MPG) after AVR, the incidence of paravalvular leak (PVL) and the need for a permanent pacemaker implantation (PPI). The secondary outcomes included aortic cross-clamp (ACC) and cardiopulmonary bypass $(\mathrm{CPB})$ times, early mortality and other postoperative complications, such as atrial fibrillation, bleeding reoperation and stroke.

Results: Eight articles were included, and all outcomes except MPG after AVR in matched valve sizes were extracted from 7 studies (RD group $=842$ patients and SU group =1,386 patients). The pooled analysis demonstrated a lower MPG in the RD group than in the SU group, with mean difference (MD) of $2.64 \mathrm{mmHg}$. The pooled risk ratios of any PVL and grade $\geq 2$ (or moderate) PVL were not significantly different between the groups; however, the incidence of PPI was significantly lower in the RD group than in the SU group, with a risk ratio of 0.69. The pooled analyses showed that the ACC and CPB times were significantly longer in the RD group than in the SU group, with weighted MDs of $8.74(\mathrm{P}<0.001)$ and 9.94 $(\mathrm{P}<0.001)$ minutes, respectively. The risks of early mortality and other postoperative complications were not significantly different between the groups.

Conclusions: AVR using RD valve was associated with better valve hemodynamics in terms of the MPG than AVR using SU valve, and better outcomes were observed in the RD group regarding PPI. Procedural times were longer in AVR using RD valve than SU valve. Early clinical outcomes showed no difference between RD and SU valve.

Keywords: Aortic valve replacement (AVR); rapid deployment valve (RD valve); sutureless valve (SU valve); metaanalysis

Submitted Dec 20, 2020. Accepted for publication Feb 26, 2021.

doi: $10.21037 /$ jtd-20-3548

View this article at: http://dx.doi.org/10.21037/jtd-20-3548

\footnotetext{
^ ORCID: Suk Ho Sohn, 0000-0001-7391-3415; Yoonjin Kang, 0000-0002-2528-173X; Ji Seong Kim, 0000-0003-2908-7130; Jae Woong Choi, 0000-0002-0921-756X; Myoung-Jin Jang, 0000-0001-8123-5001; Ho Young Hwang, 0000-0002-8935-8118.
} 


\section{Introduction}

Rapid deployment (RD) and sutureless (SU) valves have emerged to have the best of transcatheter aortic valve replacement (TAVR) and conventional surgical AVR $(1,2)$. They have become an attractive option for surgical AVR. Theoretically, RD and SU valves have advantages such as short operation time, ease of insertion even in limited operating fields and acceptable hemodynamic properties (3). A previous study demonstrated that AVR using RD or SU valves was associated with significantly shorter aortic crossclamp (ACC) and cardiopulmonary bypass (CPB) times (4). However, higher incidences of permanent pacemaker implantation (PPI) and paravalvular leakage (PVL) than in conventional surgical AVR remain concerns related to AVR using $\mathrm{RD}$ or $\mathrm{SU}$ valves. Although $\mathrm{RD}$ and $\mathrm{SU}$ valves have different characteristics of their own, they are now used mostly based on the surgeon's preference and the availability of the valves (5), and previous studies demonstrated surgical results for only one type of AVR or by pooling the data of RD-valve AVR (RDAVR) with SU-valve AVR (SUAVR) (6,7).

Therefore, this meta-analysis was conducted to directly compare early outcomes of AVR using the RD valve with those using the SU valve. We present the following article in accordance with the PRISIMA reporting checklist (available at http://dx.doi.org/10.21037/jtd-20-3548).

\section{Methods}

\section{Data source and literature search}

This systematic review and meta-analysis was conducted following the Preferred Reporting Items for Systematic Reviews and Meta-Analyses (PRISMA) guidelines (8). Fulltext articles comparing the results of RDAVR [Intuity Elite system (Edwards Lifesciences, Irvine, Calif, USA); the RD group] with those of SUAVR [Perceval-S (Livanova, London, UK); the SU group] were searched in the Medline, Embase, Cochrane Central Register of Controlled Trials and Web of Science databases on March 20, 2020. Only articles published in 2008 or later were considered.

The following keywords and medical subject heading (MeSH) terms were searched in Medline: (("aortic valve"[MeSH Terms] OR "heart valve prosthesis implantation"[MeSH Terms]) AND ("2008/01/01"[PDAT]: "3000"[PDAT])) OR ((((("aortic valve replacement"[Title/Abstract] OR "aortic valve prosthesis"[Title/ Abstract]) OR "aortic prosthesis"[Title/Abstract]) OR "prosthetic aortic valve"[Title/Abstract]) OR "aortic valve implantation"[Title/Abstract]) AND ("2008/01/01"[PDAT]:
"3000"[PDAT]))) AND (("sutureless surgical procedures"[MeSH Terms] AND ("2008/01/01"[PDAT]: "3000"[PDAT])) OR ((sutureless[Title/Abstract] OR rapid[Title/Abstract]) AND ("2008/01/01"[PDAT]: "3000"[PDAT]))). The search strategies for other databases were adapted from this Medline strategy.

\section{Study selection}

Studies were selected independently by two reviewers (SHS and $\mathrm{HYH}$ ) based on predefined selection criteria. The studies were selected through the following 2 levels of screening: the titles and abstracts of the retrieved studies were screened at the first level, and the full texts were reviewed at the second level. Studies were included if they met the following criteria: (I) they enrolled patients who underwent AVR, and (II) they compared the clinical outcomes of RDAVR with those of SUAVR. Duplicated publications with overlapping study populations were searched to select the most appropriate articles for this comparison based on the confounding bias and reported outcomes.

\section{Data extraction}

The study characteristics and the patients' baseline data were extracted independently by two reviewers (SHS and HYH). Data regarding study outcomes were also independently extracted by two reviewers (MJJ and HYH). For outcomes whose confounding-adjusted results were available, data were extracted from adjusted results, otherwise from unadjusted results. Any disagreements between reviewers were resolved through discussion by 3 authors (SHS, MJJ and HYH).

\section{Quality assessment}

The overall study quality was assessed independently by two reviewers (MJJ and $\mathrm{HYH}$ ) using the Risk Of Bias In Non-randomized Studies of Interventions (ROBINS-I) (9). In ROBINS-I, seven domains of risk of bias (ROB) were assessed, and the overall ROB was deemed low, moderate, serious or critical based on the assessment of each domain. Any disagreements between reviewers were resolved through discussion among 3 authors (SHS, MJJ and HYH).

\section{Statistical analysis}

The primary outcomes were the mean transvalvular pressure gradient of the aortic valve (AVMPG) after AVR, 
the need for PPI and the incidence of PVL. The secondary outcomes included ACC and CPB times, early mortality and postoperative complications such as bleeding reoperation and stroke.

Pooled estimates for the ACC and CPB times, early mortality and postoperative complications were presented as pooling results both from all study patients and from isolated AVR patients. For outcomes such as AVMPG, the need for PPI and the incidence of PVL, which might not be significantly affected by combined cardiac procedures, the results were pooled from all study patients.

Dichotomous outcomes were compared as odds ratios (ORs) or relative risks (RRs) with $95 \%$ confidence intervals (CIs). As a relative measure, ORs were used whenever adjusted ORs from a multivariable logistic regression were included for pooling; otherwise, RRs were applied. Continuous outcomes were presented as the weighted mean difference (MD) with 95\% CI. When the median and interquartile or overall range was reported, the mean values and standard deviations (SDs) were estimated as described (10). In one study (11), where the number of patients in both groups was reported for each of ACC and CPB times, the number of patients in each group for each time outcome was estimated by multiplying its total number by the proportion of patients in each group.

Statistical heterogeneity between the studies was assessed with the $\chi^{2}$ test and the $\mathrm{I}^{2}$ statistics. $\mathrm{I}^{2}$ values of $25 \%, 50 \%$ and $75 \%$ have been suggested to be indicators of low, moderate and high heterogeneity, respectively (12). A random-effects model with the DerSimonian and Laird method was used if substantial heterogeneity was found $\left(\mathrm{I}^{2}>50 \%\right)$; otherwise, a fixed-effects model was applied using the Mantel-Haenszel and inverse-variance methods for binary and continuous outcomes, respectively. Overall pooled estimates from all included studies were presented with pooled estimates from the studies that report results after isolated AVR.

Because the SU valve comes in 4 sizes (small, medium, large and extralarge) and the RD valves has 5 sizes (19, 21, 23, 25 and $27 \mathrm{~mm}$ ), AVMPG after surgery was compared between RDAVR and SUAVR with conservative matchings: a $19 \mathrm{~mm}$ $R D$ valve $v s$. a small $S U$ valve, $21 \mathrm{~mm} v s$. medium, $23 \mathrm{~mm} v s$. large and $25 \mathrm{~mm} v s$. extralarge; and with liberal matchings: $21 \mathrm{~mm} v$ s. small, $23 \mathrm{~mm} v$ s. medium, $25 \mathrm{~mm} v$ s. large and 27 $\mathrm{mm} v$ s. extralarge. In one study (13) where the SD of AVMPG in each valve size was not provided, the missing SD was substituted with the overall SD in each group.

A funnel plot and Egger's test for asymmetry were applied to assess the possibility of publication bias between the studies when at least 5 studies were included for pooling (14). All analyses were performed using $\mathrm{R}$ version 3.6.2 (meta package). Two-sided $\mathrm{P}$ values less than 0.050 were considered statistically significant.

\section{Results}

\section{Identification of studies}

Initial database searches identified 2,024 articles. Among these articles, 1,977 publications were excluded because they clearly did not fulfill the selection criteria based on the title and abstract. Full manuscripts of the remaining 47 articles were reviewed, and 8 publications $(5,11,13,15-19)$ were included in this study (Figure 1). Because two studies $(11,15)$ were from the same multinational registry, one study (11) was selected for baseline-data and outcome analyses. The other study (15) was used to extract data regarding AVMPG in each valve size because the former study did not report this result.

\section{Study characteristics and patient populations}

All 8 studies were non-randomized studies (NRSs). All outcomes except AVMPG in matched valve sizes were extracted from 7 studies $(5,11,13,16-19)$ with 2,228 patients (RD group $=842$ patients and SU group =1,386 patients); the largest study included 1418 patients (RD group and SU group $=407$ and 1,011 patients, respectively) (11) whereas only 43 patients (RD group and SU group $=27$ and 16 patients, respectively) were included in the smallest study (17) (Table 1). The average or median patient age was in the 70 s, and $59.4 \%$ of the patients were female. Five studies $(5,11,13,16,17)$ reported body surface area in each group, and it was higher in the RD group than in the $\mathrm{SU}$ group in four studies $(11,13,16,17)$. The proportion of minimally invasive approaches through mini-sternotomy or right anterior thoracotomy was reported in 4 studies $(5,11,16,18)$; in these studies, minimally invasive AVR was performed in $77.2 \%$ of the patients. Two studies $(13,17)$ included only patients who underwent isolated AVR. The proportions of isolated AVR ranged from $18.5 \%$ to $70.1 \%$ in the other 5 studies (Table 2). The distributions of the sizes of both RD and SU valves are described in Figure S1.

\section{Quality of the included studies}

Of the 7 NRSs mentioned above, five studies $(11,13,16,17,19)$ were graded as having an overall severe $\mathrm{ROB}$ due to the presence of confounding biases, because the 


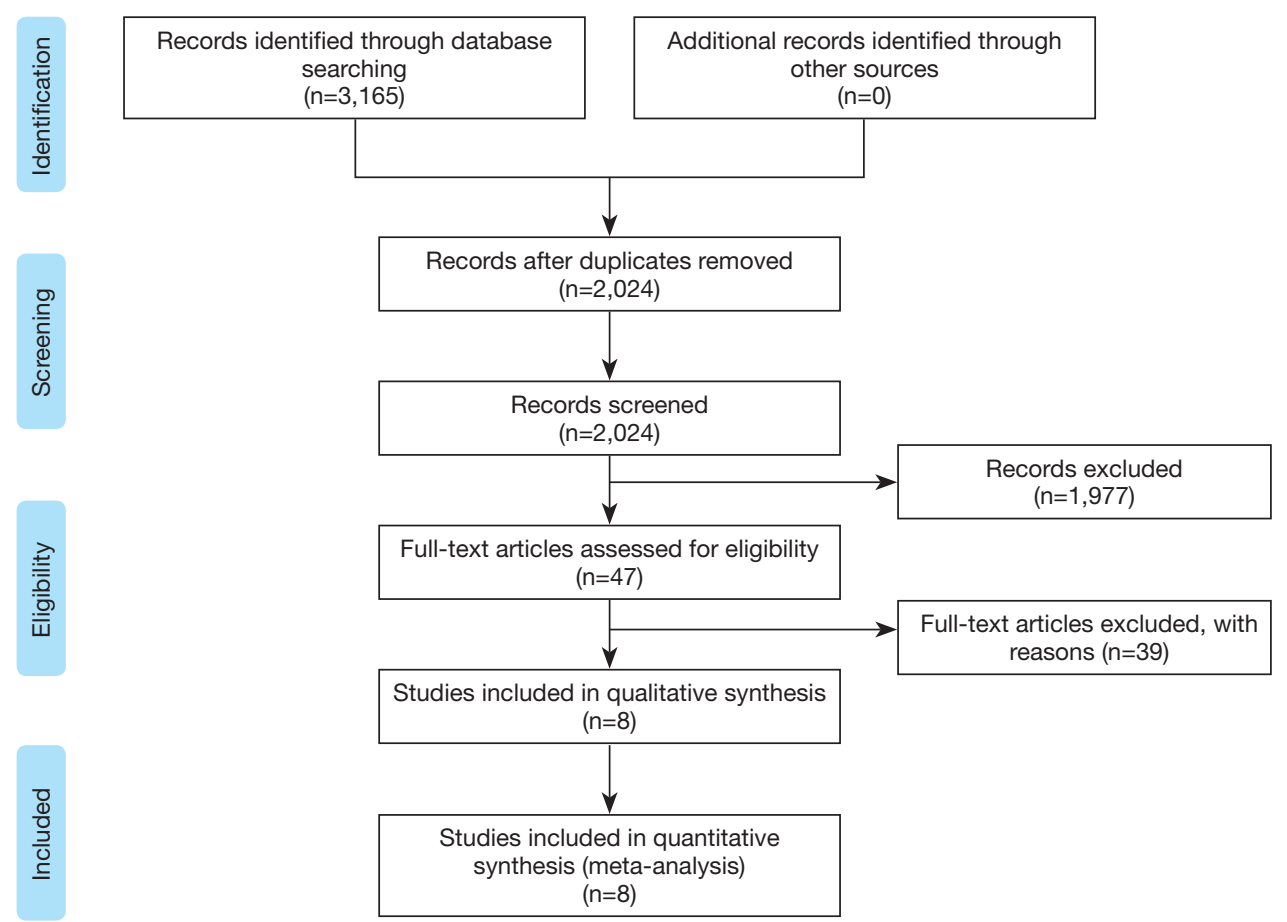

Figure 1 Preferred Reporting Items for Systematic Reviews and Meta-analyses (PRISMA) flow diagram.

Table 1 Study characteristics

\begin{tabular}{|c|c|c|c|c|c|c|c|}
\hline \multirow{2}{*}{ Study } & \multirow{2}{*}{ Operative era } & \multirow{2}{*}{ Country } & \multirow{2}{*}{$\begin{array}{l}\text { Study } \\
\text { type }\end{array}$} & \multicolumn{3}{|c|}{ Study population } & \multirow{2}{*}{ Statistical methods for adjustment } \\
\hline & & & & Total & $\mathrm{RD}$ group & SU group & \\
\hline D'Onofrio et al. & 2011-2017 & Italy & NRS & 234 & 117 & 117 & PSM \\
\hline Berretta et al. & 2007-2018 & Multi-national $^{\star}$ & NRS & 1,418 & 407 & 1,011 & $\mathrm{MVA}^{\dagger}$ \\
\hline Gotzmann et al. & 2016-2017 & Germany & NRS & 54 & 33 & 21 & None \\
\hline Ensminger et al. & 2011-2015 & Germany & NRS & 204 & 102 & 102 & PSM \\
\hline Liakopoulos et al. & 2012-2016 & Germany & NRS & 156 & 117 & 39 & None \\
\hline Jiritano et al. & Not mentioned & Italy & NRS & 43 & 27 & 16 & None \\
\hline Di Eusanio et al. ${ }^{\ddagger}$ & 2007-2017 & Multi-national $^{*}$ & NRS & 3,218 & 757 & 2,461 & None \\
\hline
\end{tabular}

*, from SURD-IR (Sutureless and Rapid Deployment Aortic Valve Replacement International Registry): Australia, Austria, Belgium, Canada, France, Germany, Italy and Switzerland. ${ }^{\dagger}$, MVA was performed in risk factor analysis for early mortality; ${ }^{\ddagger}$, because this study was from the same registry as the study by Berretta et al., it was only used to extract data regarding aortic valve mean pressure gradient in each valve size, which Berretta et al. did not report. MVA, multivariable analysis; NRS, non-randomized study; PSM, propensity score matching; RD, rapid deployment; SU, sutureless. 


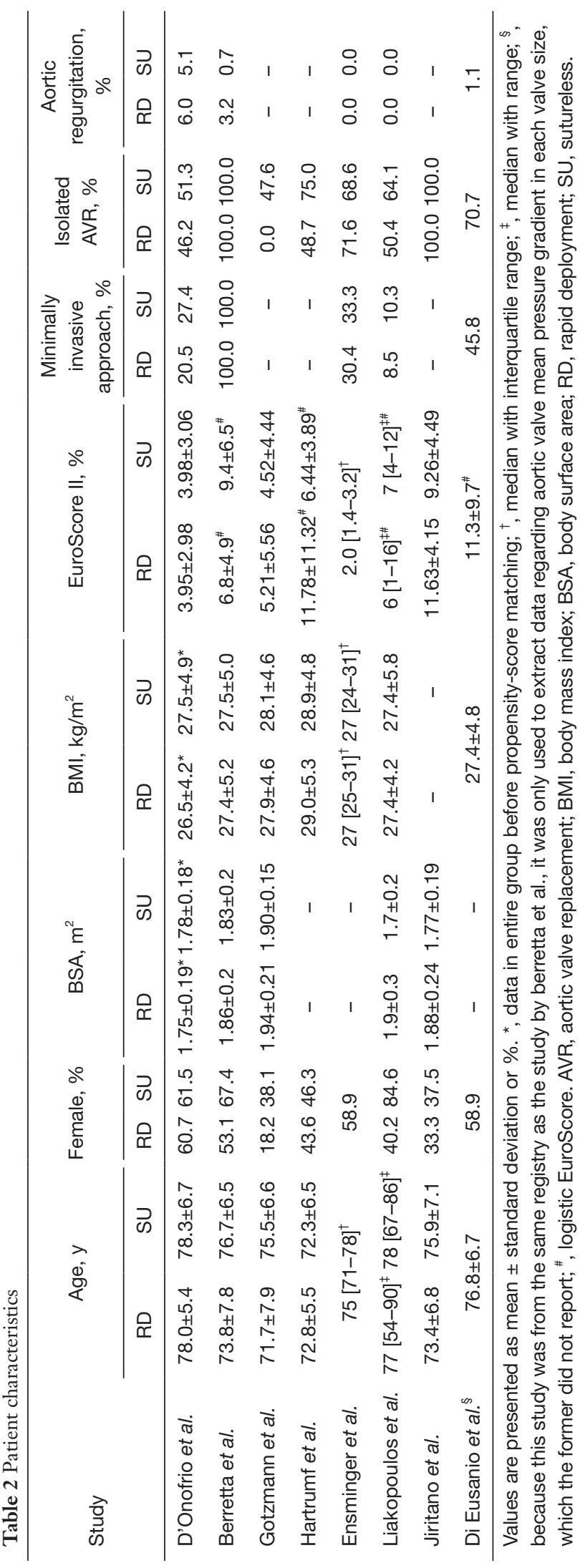

patients' baseline characteristics were not balanced across the groups and appropriate adjustments for confounding factors were not performed. Another study (15), which was used to extract data on AVMPG in matched valve sizes, also had an overall severe ROB for the same reason. The other 2 studies $(5,18)$ used propensity score matching and had an overall moderate ROB (Table S1).

\section{Mean transvalvular pressure gradient}

Six studies $(5,11,13,16,18,19)$ demonstrated AVMPG after surgery, and 5 studies $(5,13,16,18,19)$ reported the AVMPG in each valve size. One more study (15) was included in the analysis to extract data regarding AVMPG in each valve size as previously described. A pooled analysis from 6 studies with 2,820 patients demonstrated a lower AVMPG in the $\mathrm{RD}$ group than in the SU group [weighted MD (95\% CI); $-2.64(-3.74,-1.54) \mathrm{mmHg}$, Figure 2]. When the AVMPG was compared under liberal matching of valve sizes, it was still significantly lower in the RD group than in the $\mathrm{SU}$ group in all valve sizes (Figure 3). When conservative matching was used, AVMPG was significantly higher in the $19 \mathrm{~mm}$ RD valve than the small SU valve, but it was lower in the 23 and $25 \mathrm{~mm}$ RD valves than the large and extralarge SU valves, respectively (Figure 4).

\section{Risk of the need for PPI}

The risk of the need for PPI after AVR was drawn from 6 studies with 1,735 patients $(5,11,13,16,18,19)$. The pooled analysis demonstrated that the risk of PPI was significantly lower in the RD group than in the SU group [RR (95\% CI) $=0.69(0.50,0.94), \mathrm{I}^{2}=0 \%$, Figure 5$]$.

\section{Risk of $P V L$}

Pooled analyses of any PVL and grade $\geq 2$ (or moderate) PVL were drawn from 5 studies $(5,11,13,16,18)$ with 1,350 patients and from 6 studies $(5,11,13,16,18,19)$ with 1,469 patients, respectively. There were no significant differences in the risk of any PVL $[R R(95 \% \mathrm{CI})=0.60(0.28$, 1.30), $\mathrm{I}^{2}=65.9 \%$ ) or grade $\geq 2$ (or moderate) PVL [RR ( $95 \%$ CI) $\left.=0.83(0.31,2.18), I^{2}=0.0 \%\right]$ between the RD and $\mathrm{SU}$ groups (Figure 6).

\section{$A C C$ and $C P B$ times}

All 7 studies $(5,11,13,16-19)$ demonstrated results compared 


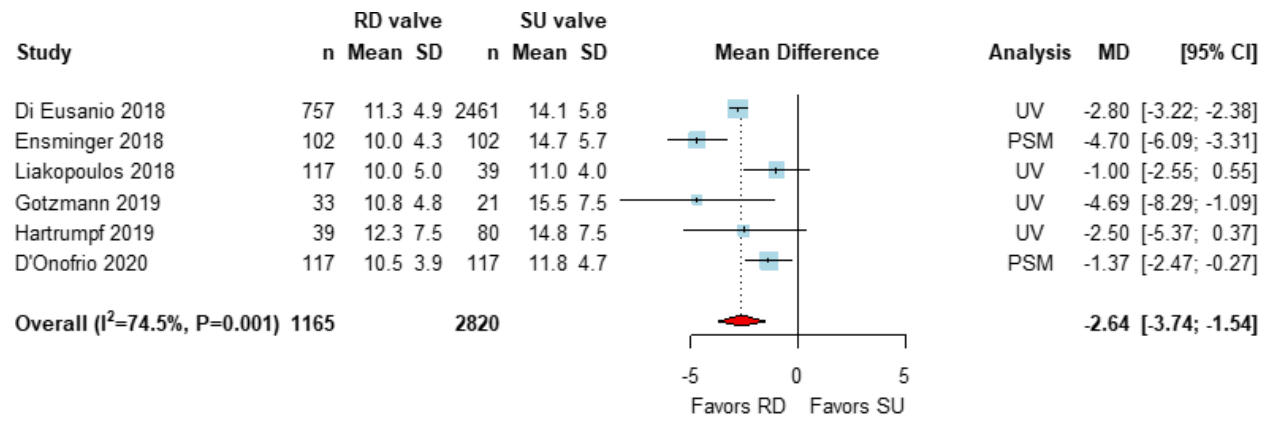

Figure 2 Weighted mean difference (MD) of the mean transvalvular pressure gradient (mmHg) in rapid deployment (RD) versus sutureless (SU) aortic valve replacement. CI, confidence interval; PSM, propensity score matching; SD, standard deviation; UV, univariate.

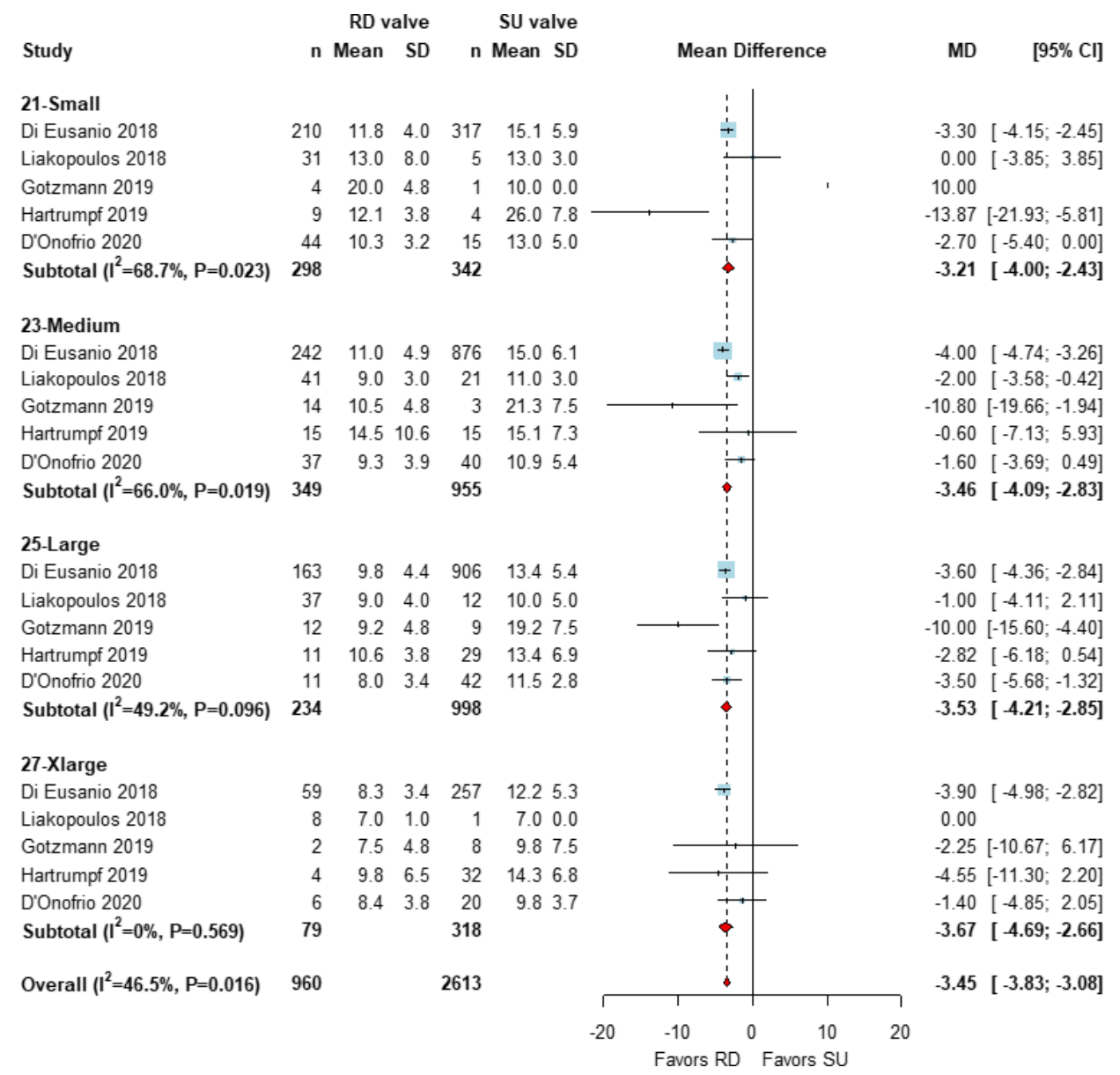

Figure 3 Weighted mean differences (MDs) of transvalvular mean pressure gradient in rapid deployment (RD) versus sutureless (SU) aortic valve replacement by matching RD with SU valve sizes as $21 \mathrm{~mm}$ with small, $23 \mathrm{~mm}$ with medium, $25 \mathrm{~mm}$ with large and $27 \mathrm{~mm}$ with extralarge. CI, confidence interval; SD, standard deviation. 


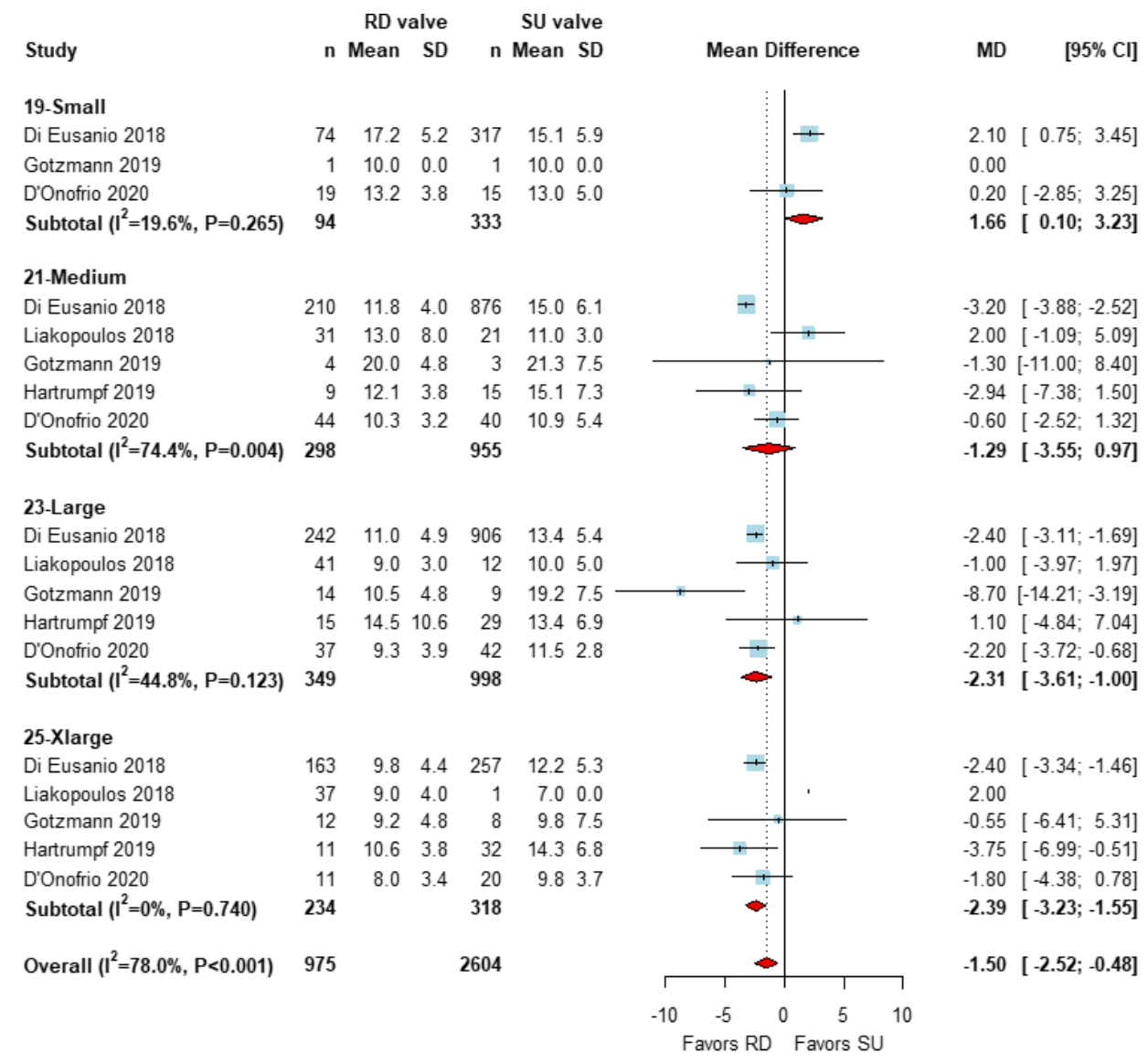

Figure 4 Weighted mean differences (MDs) of transvalvular mean pressure gradient in rapid deployment (RD) versus sutureless (SU) aortic valve replacement by matching RD with SU valve sizes as $19 \mathrm{~mm}$ with small, $21 \mathrm{~mm}$ with medium, $23 \mathrm{~mm}$ with large and $25 \mathrm{~mm}$ with extralarge. CI, confidence interval; SD, standard deviation.

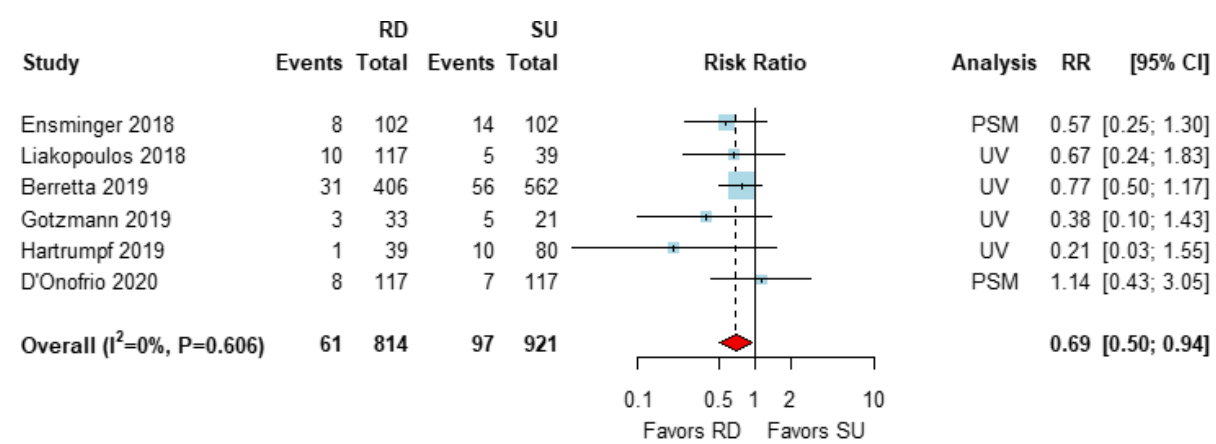

Figure 5 Risk ratios (RRs) of permanent pacemaker implantation in rapid deployment (RD) versus sutureless (SU) aortic valve replacement. CI, confidence interval; PSM, propensity score matching; SD, standard deviation; UV, univariate. 


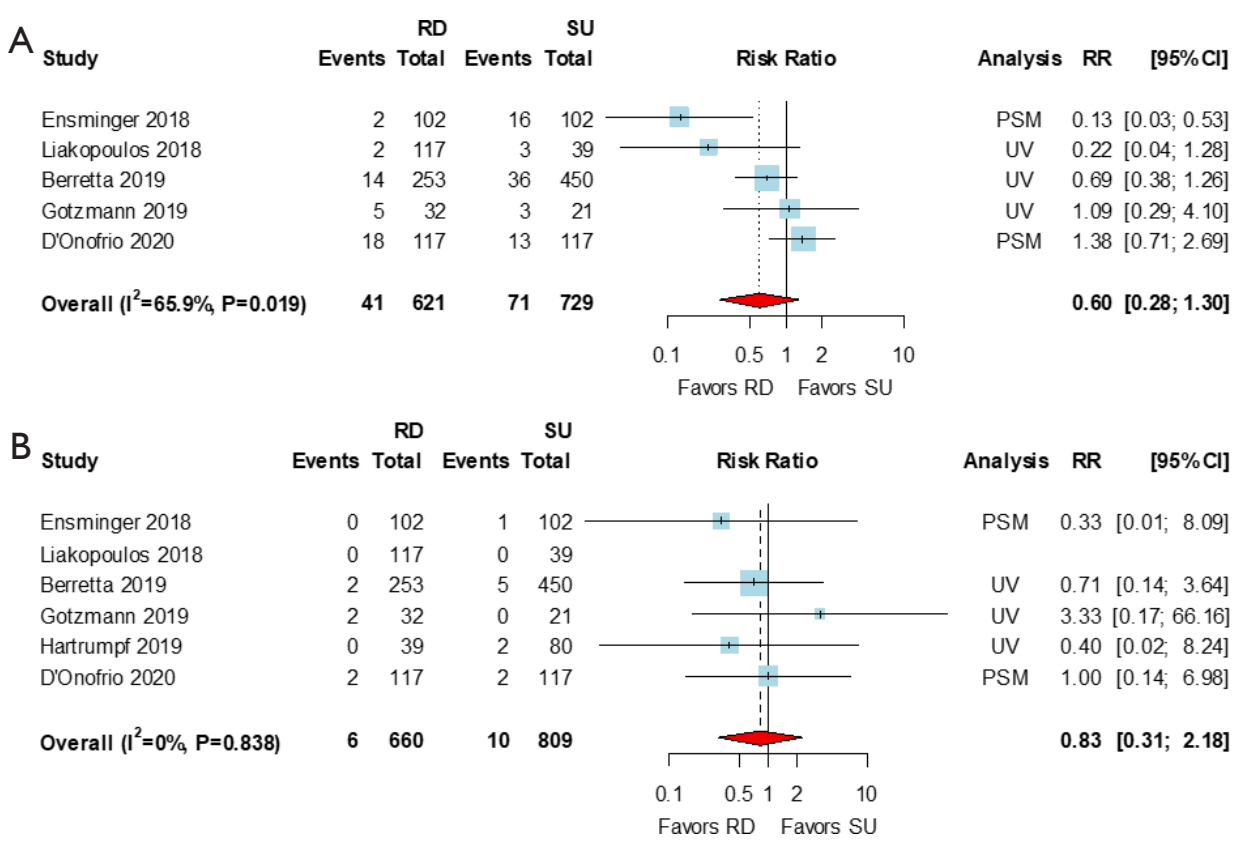

Figure 6 Risk ratios (RRs) of (A) any paravalvular leak and (B) a paravalvular leak of grade $\geq 2$ (or moderate) in rapid deployment (RD) versus sutureless (SU) aortic valve replacement. CI, confidence interval; PSM, propensity score matching; SD, standard deviation; UV, univariate.

the ACC and CPB times between the 2 groups. Pooled analyses using a random-effects model revealed that the ACC and CPB times were longer in the RD group than in the SU group [weighted MD (95\% CI): 8.74 (3.25, 14.23) minutes, $\mathrm{P}<0.001$ and 9.94 (2.23, 17.65) minutes, $\mathrm{P}<0.001$, respectively]. These results were similar when the ACC and CPB times were compared in patients who underwent isolated AVR from 6 studies [weighted MD $(95 \%$ CI): 6.24 (1.96, 10.52) minutes, $\mathrm{P}<0.001$ and 7.95 (1.27, 14.63) minutes, respectively] (Figure 7).

\section{Risks of early mortality and postoperative complications}

Early mortality was reported in all studies, but the RRs could not be calculated in one study (17), in which the early mortality rate in both groups was $0 \%$. Early mortality was defined as 30-day mortality and in-hospital death in three $(5,13,16)$ and another three $(11,18,19)$ studies, respectively. The risk of early mortality was not significantly different between the RD and SU groups in the overall patient sample or in isolated AVR patients [RR $(95 \% \mathrm{CI})=1.13(0.53,2.40)$, $\mathrm{I}^{2}=0 \%$ and $0.77(0.28,2.16), \mathrm{I}^{2}=0 \%$, respectively, Figure S2]. The risks of bleeding reoperation and stroke were reported in $5(11,13,16,17,19)$ and all 7 studies, respectively. The pooled analyses demonstrated no significant differences in the risks of these postoperative complications between the 2 groups, either among the overall patients or among isolated AVR patients (Figures S3,S4).

\section{Publication bias}

There was no evidence of publication bias in the funnel plots of the outcomes. Egger's tests also indicated that publication bias was insignificant for each outcome (Figure S5).

\section{Discussion}

The present study demonstrated 2 main findings. First, overall AVMPG was lower after RDAVR than after SUAVR. Second, the need for PPI was lower after RDAVR than after SUAVR.

TAVR was initially developed to treat patients who had a high or prohibitive risk for surgical AVR. However, the indications of TAVR have rapidly expanded to patients with moderate and even low surgical risk $(20,21)$. The advancements in RD and SU valves have taken their lead from the advantages of TAVR, such as a self- or balloonexpandable nature and no or less need for sutures and knottying; these technologies have several advantages, such as a short operation time, ease of insertion by avoiding suturing 


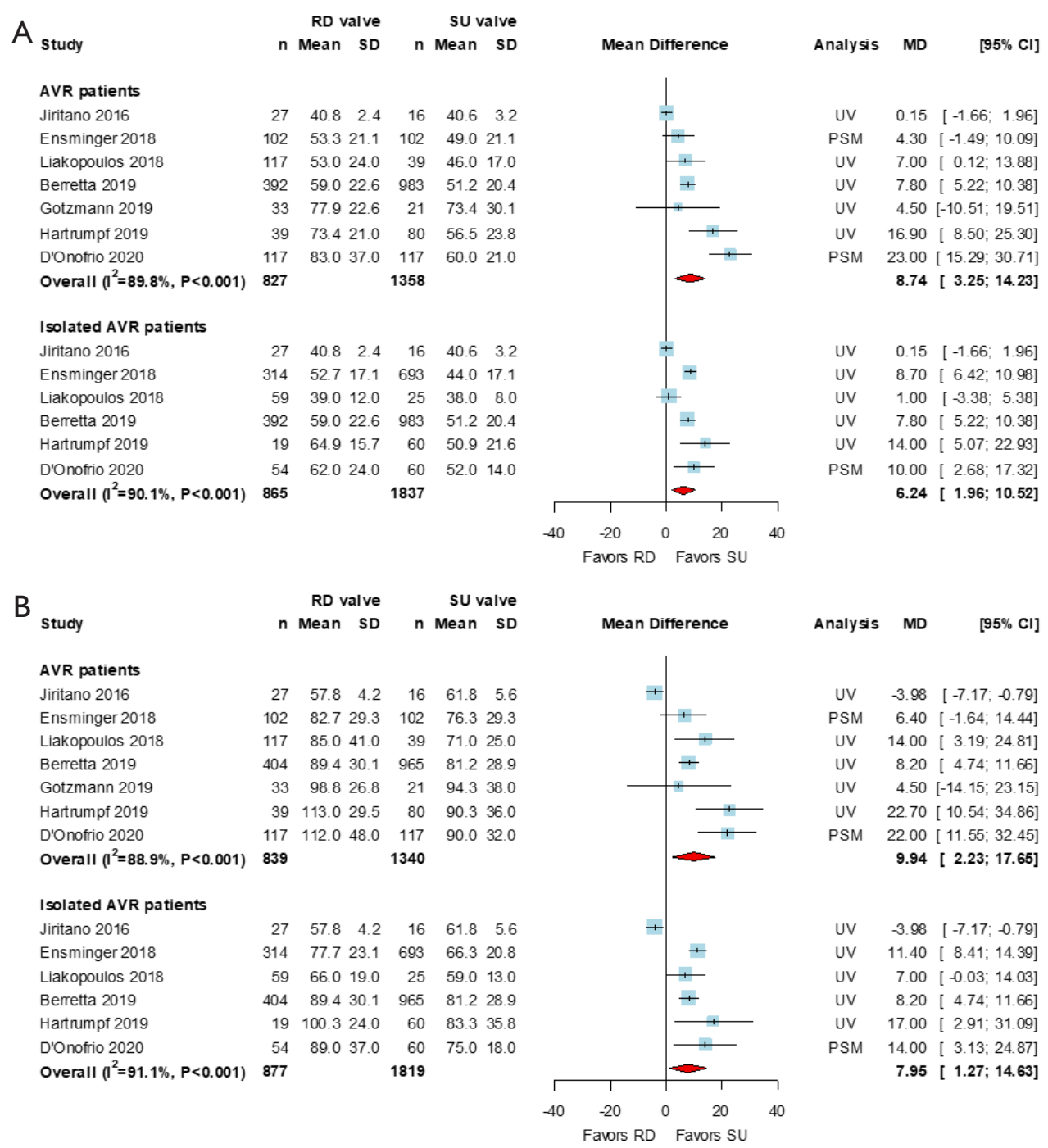

Figure 7 Weighted mean differences (MDs) of (A) aortic cross-clamp and (B) cardiopulmonary bypass times in rapid deployment (RD) versus sutureless (SU) aortic valve replacement (AVR). Pooled analyses from overall patients and from isolated AVR patients are presented. CI, confidence interval; PSM, propensity score matching; SD, standard deviation; UV, univariate.

and knot-tying even in limited operating fields, and favorable hemodynamic properties (3). However, the risks of PPI and PVL are still concerns related to AVR using RD or SU valves, as with TAVR (22).

The present meta-analysis showed that AVMPG after surgery was satisfactory in both the RD and SU groups, with mean AVMPG of 10.72 and $13.36 \mathrm{mmHg}$, respectively. Theoretically, the SU valve could provide a larger effective orifice area than the RD valve because it does not have any valve stent. However, the present study showed that the AVMPG was lower after RDAVR than after SUAVR; although the mean difference between the 2 groups was only $2.64 \mathrm{mmHg}$, it was statistically significant in the pooled analysis. In addition, the AVMPG was lower in the RD group than in the SU group in all liberal matchings. Even under conservative matching, the AVMPG was lower in the 23 and $25 \mathrm{~mm} \mathrm{RD}$ valves than in the large and extralarge SU valves, respectively, whereas it was higher in the $19 \mathrm{~mm}$ $\mathrm{RD}$ valve than in the small SU valve. Because the AVMPG is largely influenced by demographic factors, particularly body surface area, there could be a bias in this result of the present study. However, the impact of this bias on the study results might be minimal since the patients in the SU group had a smaller BSA in general and the SU valves used in 
the enrolled study were similar in size to or larger than the $\mathrm{RD}$ valves. A possible explanation for this finding might be that the actual effective orifice area could be smaller than generally expected after SUAVR if the self-expansion of the $\mathrm{SU}$ valve is not completely achieved due to technical error or oversizing (23). In contrast, the skirt of the RD valve might optimize the flow characteristics through the valve inlet by widening and reshaping the LV outflow tract, and it might lead to more laminar blood flow across the prosthesis by limiting the active constriction of the $\mathrm{LV}$ outflow tract during systole, thereby contributing to better hemodynamic results of the valve (24-27).

Both RD and SU valves carry the risks of PVL and conduction disturbance compared with conventional AVR because neither valve needs circumferential sutures to be secured, and each could interfere with the conduction pathway by its own mechanisms. The RD valve has a distinctive 'skirt' in its structure and can compress the conduction, which causes postoperative conduction abnormality. Distorted native aortic annulus and incomplete fitting of the $\mathrm{RD}$ valve to the annulus would result in complete atrioventricular block as well. On the other hand, the SU valve has a rather planar supra-annular and intraannular sealing collar, which is intended to land on the semilunar aortic annulus. Thus, a trivial misplacement of the inflow ring would easily lead to the interruption of normal conduction. A lower level of implantation, which results in compression of conduction pathway during subannular expansion, is also thought to be associated with the occurrence of heart block $(28,29)$. In the present metaanalysis, the risk of PPI after surgery was significantly lower in the RD group than in the SU group, but the risk of PVL was similar between the 2 groups.

Theoretically, the difference of PPI rate between RD and SU group would be explained by the followings: after AVR is performed, the pressure gradient is decreased, the left ventricular hypertrophy regresses gradually, and myocardial edema also resolved in the postoperative period. Because the skirt of the RD valve becomes a fixed structure with no more outward force after the balloon expansion is completed, the conduction status in the RD group has the chance of recovery. However, the $\mathrm{SU}$ valve has a self-expanding nature and will constantly compress the conduction pathway with a persistent outward force (30). Clinically, Coti et al. (31) reported that preoperative right bundle branch block and concomitant procedures were the independent predictors for new pacemaker implantation after RDAVR.

Despite significant differences in the primary outcomes, these differences did not translate into differences in secondary outcomes, such as early mortality, bleeding reoperation and stroke.

Some surgeons would choose the RD or SU valve to reduce ACC time for high-risk patients because shortening ACC time by several minutes could be of paramount importance to the outcome. A previous study (32) retrospectively analyzed approximately 1,000 patients who underwent surgical AVR and showed that the ACC time was an independent predictor of cardiovascular morbidity after surgery, with a $1.4 \%$ increase in the risk per oneminute increase in ACC time. The present study showed that the use of the SU valve could further shorten the ACC time by 6 minutes, although this result should be interpreted cautiously because the gained experience of surgeons is greater with $S U$ valve than with $R D$ valve and the proportion of minimally invasive procedures was different between the studies. This result might be expected because the SU valve does not need knot-tying or balloon inflation, whereas the RD valve needs knot-tying of at least 3 sutures, and manipulation of the balloon system and balloon inflation for 10 seconds are also mandatory. Because the procedural time of 'rapid deployment' valve is not faster than that of SU valve, opponents might argue whether the terminology "rapid deployment" could be really justified. In addition, aortotomy on the more distal part of the ascending aorta in SUAVR might make aortotomy repair easier than in the case of RDAVR. Therefore, the SU valve could be more beneficial for the treatment of high-risk patients in whom shortening ACC time is critical to the outcome.

In contrast, other surgeons might think that it is controversial whether shortening ACC time by several minutes would indeed affect the clinical outcomes of AVR. The ACC time of RD and SU AVR from the studies included in this meta-analysis was around 40-60 minutes while that of isolated primary surgical AVR from the Society of Thoracic Surgeons (STS) Adult Cardiac Surgery Database was around 70 minutes (33). Compared with this large international registry, it is not conclusive that the early clinical outcomes of RD and SU valves from this metaanalysis are superior to those of conventional valves. Thus, reduction of several minutes in the procedural times might be regarded as not very competitive to some surgeons.

A meta-analysis (34) comparing results after the RD and SU valves was recently published while the present study was being prepared. There were some differences in included studies and total number of patients between that study and ours. It might be due to the differences in 
inclusion criteria such as the duplication, and data extraction strategy in studies where propensity score matching was performed. Most of all, it would be novel and distinctive that we compared the mean transvalvular pressure gradient between the groups based on the prosthesis size, considering two possible matchings.

There are several limitations of the present study that should be noted. First, only seven studies were included in the present meta-analysis. Second, all included studies were NRSs, and only 2 of the 7 studies reported adjusted results. Third, the SURD-IR (Sutureless and Rapid Deployment Aortic Valve Replacement International Registry) was the largest contribution to this analysis and the results are in the line with these published results. Fourth, regarding that the number of patients who underwent AVR using SU valve up to date worldwide is clearly higher than that of $\mathrm{RD}$ valve, procedural times could be biased by the gained experience of surgeons. The fact that the proportions of minimally invasive approaches and combined procedures were different between the studies would also confound the outcomes regarding procedural times. Finally, a publication bias could not be ruled out, although the funnel plots and Egger's test indicated that the publication bias was insignificant in all results.

In conclusion, valve hemodynamics in terms of the mean transvalvular pressure gradient were significantly better in RDAVR than in SUAVR, and better outcomes were observed in RDAVR regarding PPI, while procedural times were shorter in SUAVR than in RDAVR. The RD valve and $\mathrm{SU}$ valve have strengths and weaknesses when they are compared and contrasted with one another, although they share many things in common in terms of their indications and their clinical applications. Thus, deliberate and considered selection of the RD versus the $\mathrm{SU}$ valve will be required for each individual patient.

\section{Acknowledgments}

The authors thank Dr. Jae Suk Yoo (Sejong General Hospital, Bucheon, Korea) and Soonchang Hong (Wonju Severance Christian Hospital, Wonju, Korea) for providing valuable comments on the interpretation of the study results.

Funding: None.

\section{Footnote}

Reporting Checklist: The authors have completed the
PRISMA reporting checklist. Available at http://dx.doi. org/10.21037/jtd-20-3548

Peer Review File: Available at http://dx.doi.org/10.21037/jtd20-3548

Conflicts of Interest: All authors have completed the ICMJE uniform disclosure form (available at http://dx.doi. org/10.21037/jtd-20-3548). The authors have no conflicts of interest to declare.

Ethical Statement: The authors are accountable for all aspects of the work in ensuring that questions related to the accuracy or integrity of any part of the work are appropriately investigated and resolved.

Open Access Statement: This is an Open Access article distributed in accordance with the Creative Commons Attribution-NonCommercial-NoDerivs 4.0 International License (CC BY-NC-ND 4.0), which permits the noncommercial replication and distribution of the article with the strict proviso that no changes or edits are made and the original work is properly cited (including links to both the formal publication through the relevant DOI and the license). See: https://creativecommons.org/licenses/by-nc-nd/4.0/.

\section{References}

1. Schlömicher M, Haldenwang PL, Moustafine V, et al. Minimal access rapid deployment aortic valve replacement: initial single-center experience and 12-month outcomes. J Thorac Cardiovasc Surg 2015;149:434-40.

2. Shrestha M, Maeding I, Höffler K, et al. Aortic valve replacement in geriatric patients with small aortic roots: are sutureless valves the future? Interact Cardiovasc Thorac Surg 2013;17:778-82.

3. Shalabi A, Spiegelstein D, Sternik L, et al. Sutureless Versus Stented Valve in Aortic Valve Replacement in Patients With Small Annulus. Ann Thorac Surg 2016;102:118-22.

4. Sohn SH, Jang MJ, Hwang HY, et al. Rapid deployment or sutureless versus conventional bioprosthetic aortic valve replacement: A meta-analysis. J Thorac Cardiovasc Surg 2018;155:2402-12.e5.

5. D'Onofrio A, Salizzoni S, Filippini C, et al. Surgical aortic valve replacement with new-generation bioprostheses: Sutureless versus rapid-deployment. J Thorac Cardiovasc Surg 2020;159:432-42.e1. 
6. Santarpino G, Berretta P, Fischlein T, et al. Operative outcome of patients at low, intermediate, high and 'very high' surgical risk undergoing isolated aortic valve replacement with sutureless and rapid deployment prostheses: results of the SURD-IR registry. Eur J Cardiothorac Surg 2019;56:38-43.

7. Smith AL, Shi WY, Rosalion A, et al. Rapid-deployment versus conventional bio-prosthetic aortic valve replacement. Heart Lung Circ 2017;26:187-93.

8. Moher D, Liberati A, Tetzlaff J, et al. Preferred reporting items for systematic reviews and meta-analyses: The prisma statement. Int J Surg 2010;8:336-41.

9. Sterne JA, Hernán MA, Reeves BC, et al. Robins-I: A tool for assessing risk of bias in non-randomised studies of interventions. BMJ 2016;355:i4919.

10. Wan X, Wang W, Liu J, et al. Estimating the sample mean and standard deviation from the sample size, median, range and/or interquartile range. BMC Medical Research Methodology 2014;14:135.

11. Berretta P, Andreas M, Carrel TP, et al. Minimally invasive aortic valve replacement with sutureless and rapid deployment valves: a report from an international registry (Sutureless and Rapid Deployment International Registry). Eur J Cardiothorac Surg 2019;56:793-9.

12. Higgins JP, Thompson SG, Deeks JJ, et al. Measuring inconsistency in meta-analyses. BMJ 2003;327:557-60.

13. Gotzmann M, Wilbring M, Charitos E, et al. Hemodynamic Comparison of Sutureless and RapidDeployment Valves with Conventional Bioprostheses. Thorac Cardiovasc Surg 2020;68:584-94.

14. Duval S, Tweedie R. Trim and fill: A simple funnel-plotbased method of testing and adjusting for publication bias in meta-analysis. Biometrics 2000;56:455-63.

15. Di Eusanio M, Phan K, Berretta P, et al. Sutureless and Rapid-Deployment Aortic Valve Replacement International Registry (SURD-IR): early results from 3343 patients. Eur J Cardiothorac Surg 2018;54:768-73.

16. Liakopoulos OJ, Gerfer S, Weider S, et al. Direct Comparison of the Edwards Intuity Elite and Sorin Perceval S Rapid Deployment Aortic Valves. Ann Thorac Surg 2018;105:108-14.

17. Jiritano F, Cristodoro L, Malta E, et al. Thrombocytopenia after sutureless aortic valve implantation: Comparison between Intuity and Perceval bioprostheses. J Thorac Cardiovasc Surg 2016;152:1631-3.

18. Ensminger S, Fujita B, Bauer T, et al. Rapid Deployment Versus Conventional Bioprosthetic Valve Replacement for Aortic Stenosis. J Am Coll Cardiol 2018;71:1417-28.
19. Hartrumpf M, Kuehnel RU, Schroeter F, et al. Clinical Short-Term Outcome and Hemodynamic Comparison of Six Contemporary Bovine Aortic Valve Prostheses. Thorac Cardiovasc Surg 2020;68:557-66.

20. Mack MJ, Leon MB, Thourani VH, et al. Transcatheter Aortic-Valve Replacement with a Balloon-Expandable Valve in Low-Risk Patients. N Engl J Med 2019;380:1695-705.

21. Popma JJ, Deeb GM, Yakubov SJ, et al. Transcatheter Aortic-Valve Replacement with a Self-Expanding Valve in Low-Risk Patients. N Engl J Med 2019;380:1706-15.

22. Sohn SH, Hwang HY. Permanent pacemaker implantation is never a benign complication after aortic valve replacement. J Thorac Dis 2018;10:S4080-1.

23. Cerillo AG, Amoretti F, Mariani M, et al. Increased Gradients After Aortic Valve Replacement With the Perceval Valve: The Role of Oversizing. Ann Thorac Surg 2018;106:121-8.

24. Haverich A, Wahlers TC, Borger MA, et al. Threeyear hemodynamic performance, left ventricular mass regression, and prosthetic-patient mismatch after rapid deployment aortic valve replacement in 287 patients. J Thorac Cardiovasc Surg 2014;148:2854-60.

25. Borger MA, Dohmen PM, Knosalla C, et al. Haemodynamic benefits of rapid deployment aortic valve replacement via a minimally invasive approach: 1 -year results of a prospective multicentre randomized controlled trial. Eur J Cardiothorac Surg 2016;50:713-20.

26. Ai L, Chen H, Lin V, et al. Rapid Deployment Aortic Valves Deliver Superior Hemodynamic Performance In Vitro. Innovations (Phila) 2017;12:338-45.

27. Sadri V, Bloodworth CH 4th, Madukauwa-David ID, et al. A mechanistic investigation of the EDWARDS INTUITY Elite valve's hemodynamic performance. Gen Thorac Cardiovasc Surg 2020;68:9-17.

28. Yanagawa B, Cruz J, Boisvert L, et al. A simple modification to lower incidence of heart block with sutureless valve implantation. J Thorac Cardiovasc Surg 2016;152:630-2.

29. Bouhout I, Mazine A, Rivard L, et al. Conduction Disorders After Sutureless Aortic Valve Replacement. Ann Thorac Surg 2017;103:1254-60.

30. Villa E, Clerici A, Messina A, et al. Risk Factors for Permanent Pacemaker after Implantation of Surgical or Percutaneous Self-Expanding Aortic Prostheses. J Heart Valve Dis 2016;25:663-71.

31. Coti I, Schukro C, Drevinja F, et al. Conduction disturbances following surgical aortic valve replacement 
with a rapid-deployment bioprosthesis. J Thorac

Cardiovasc Surg 2020;S0022-5223(20)30433-5.

32. Ranucci M, Frigiola A, Menicanti L, et al. Aortic crossclamp time, new prostheses, and outcome in aortic valve replacement. J Heart Valve Dis 2012;21:732-9.

33. Thourani VH, Suri RM, Gunter RL, et al. Contemporary real-world outcomes of surgical aortic valve replacement in
141,905 low-risk, intermediate-risk, and high-risk patients. Ann Thorac Surg 2015;99:55-61.

34. Flynn CD, Williams ML, Chakos A, et al. Sutureless valve and rapid deployment valves: a systematic review and meta-analysis of comparative studies. Ann Cardiothorac Surg 2020;9:364-74.

Cite this article as: Sohn SH, Kang Y, Kim JS, Choi JW, Jang MJ, Hwang HY. Direct comparison of rapid deployment versus sutureless aortic valve replacement: a meta-analysis. J Thorac Dis 2021;13(4):2203-2215. doi: 10.21037/jtd-20-3548 


\section{Supplementary}

Table S1 Quality Assessment by Risk Of Bias In Non-randomized Studies of Interventions (ROBINS-I)

\begin{tabular}{|c|c|c|c|c|c|c|c|c|}
\hline Study & $\begin{array}{l}\text { Bias due to } \\
\text { confounding }\end{array}$ & $\begin{array}{l}\text { Bias in selection } \\
\text { of participants } \\
\text { into the study }\end{array}$ & $\begin{array}{l}\text { Bias in } \\
\text { measurement of } \\
\text { interventions }\end{array}$ & $\begin{array}{l}\text { Bias due to } \\
\text { departures } \\
\text { from intended } \\
\text { interventions }\end{array}$ & $\begin{array}{l}\text { Bias due to } \\
\text { missing data }\end{array}$ & $\begin{array}{c}\text { Bias in } \\
\text { Measurement } \\
\text { of outcomes }\end{array}$ & $\begin{array}{c}\text { Bias in selection } \\
\text { of the reported } \\
\text { result }\end{array}$ & Overall \\
\hline D'Onofrio et al. & Moderate & Low & Low & Low & Low & Low & Low & Moderate \\
\hline Berretta et al. & $\begin{array}{c}\text { Serious/ } \\
\text { moderate* }^{*}\end{array}$ & Low & Low & Low & Low & Low & Low & $\begin{array}{c}\text { Serious/ } \\
\text { moderate* }\end{array}$ \\
\hline Hartrumf et al. & Serious & Low & Low & Low & Low & Low & Low & Serious \\
\hline Ensminger et al. & Moderate & Low & Low & Low & Low & Low & Low & Moderate \\
\hline Liakopoulos et al. & Serious & Low & Low & Low & Low & Low & Low & Serious \\
\hline
\end{tabular}

* Multivariable analysis was performed in the risk factor analysis for early mortality. ${ }^{\dagger}$ Assessed for the outcomes regarding mean transvalvular pressure gradient of the aortic valve in each valve size.

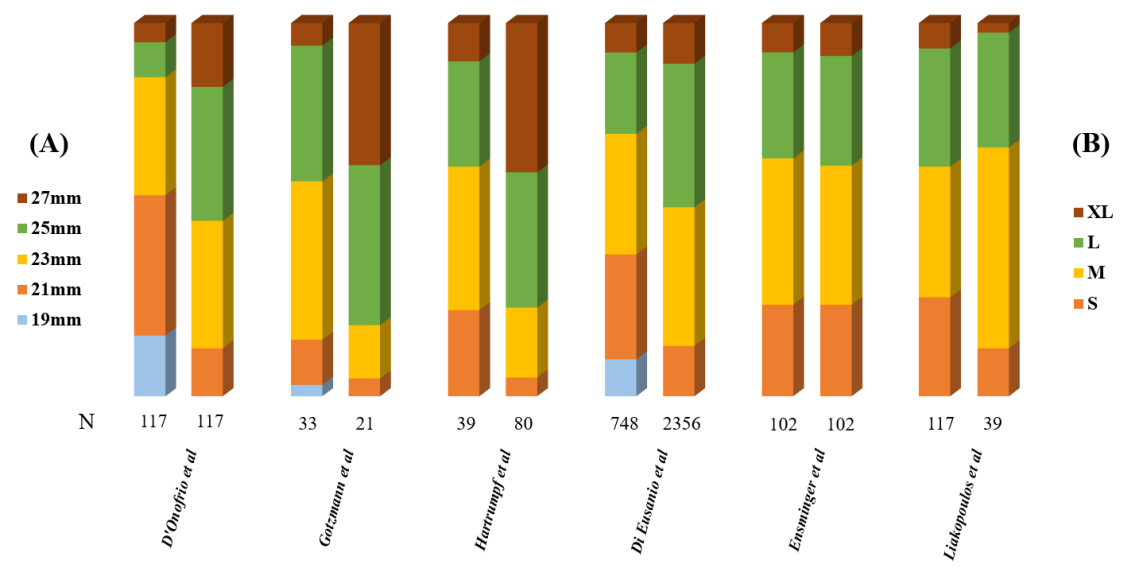

Figure S1 The distribution of the sizes of both (A) rapid deployment and (B) sutureless valves used in the included studies. S, small; M, medium; L, large; XL, extralarge. 


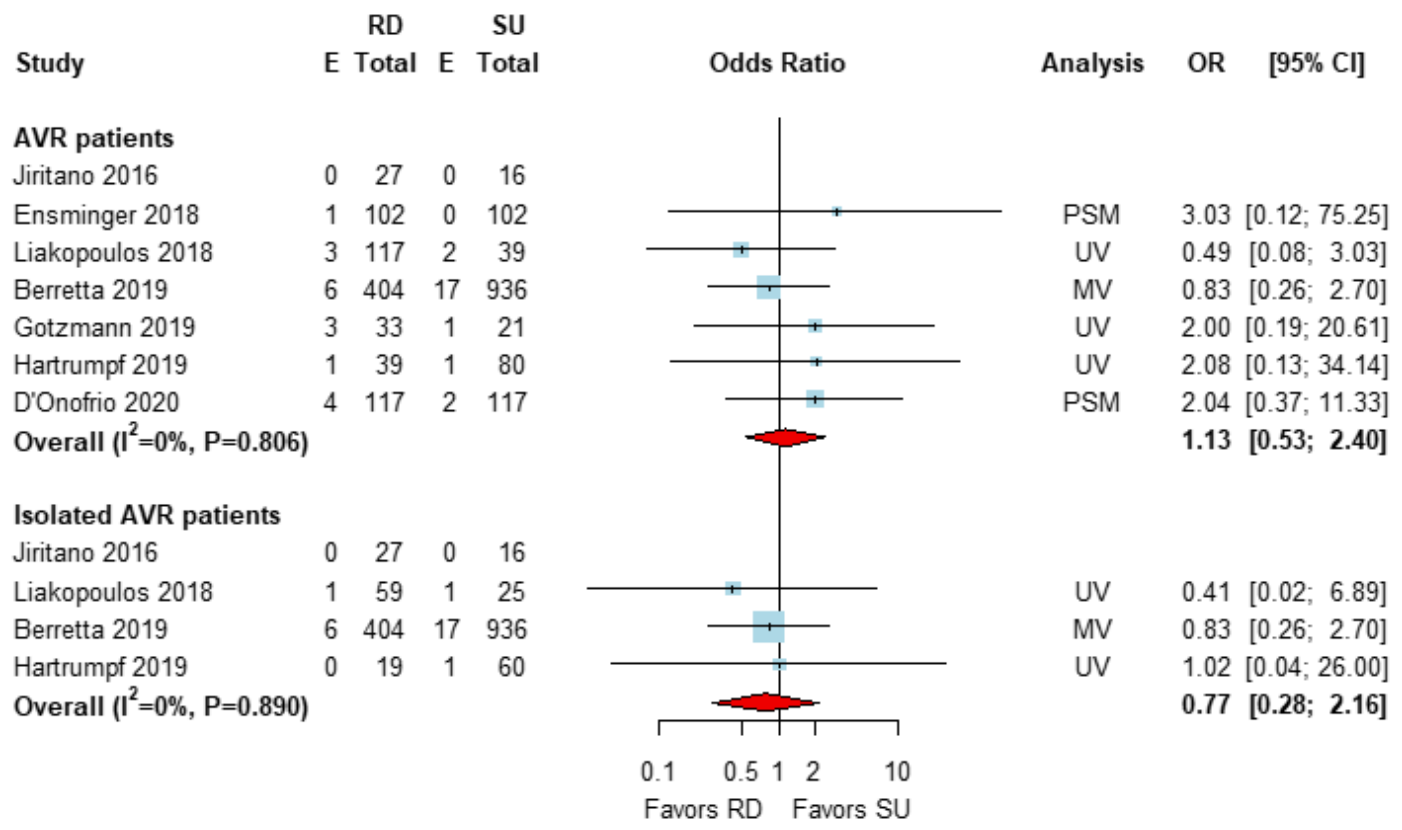

Figure S2 Odds ratios (ORs) of early mortality in rapid deployment (RD) versus sutureless (SU) aortic valve replacement (AVR). Pooled analyses from overall patients and from isolated AVR patients are presented. CI, confidence interval; MV, multivariable; PSM, propensity score matching; UV, univariate.

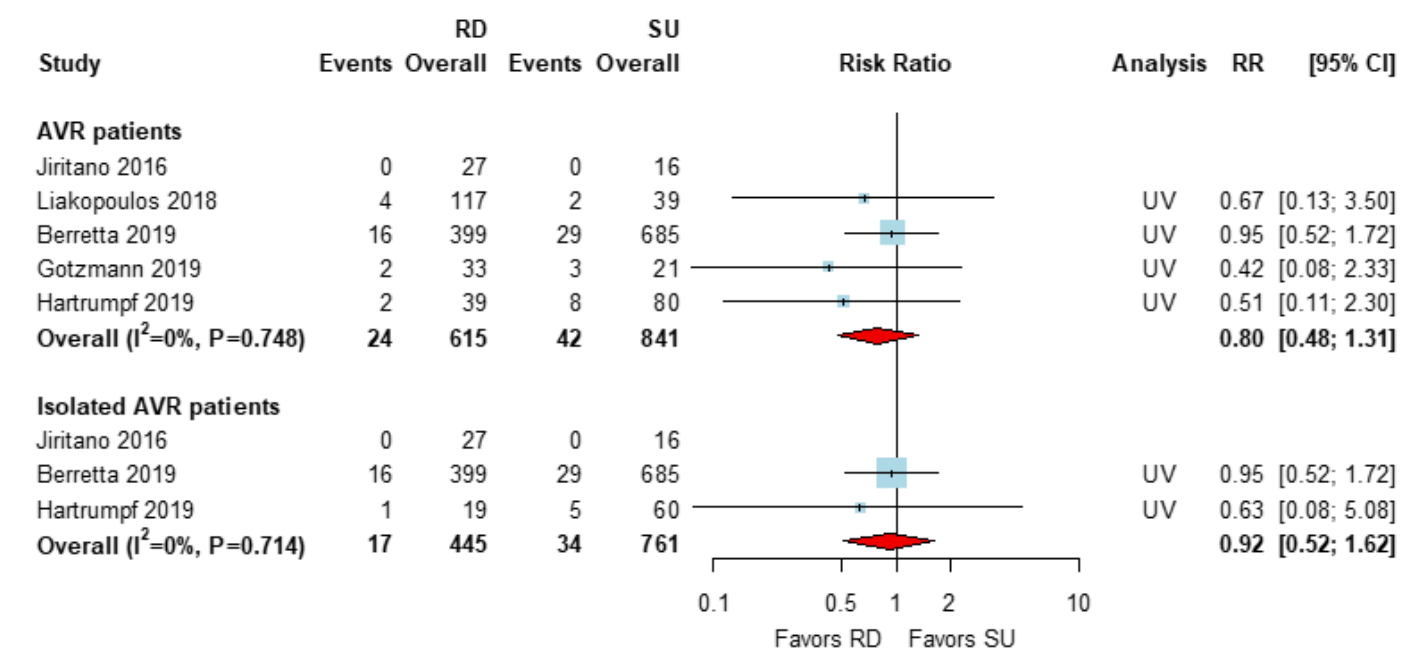

Figure S3 Risk ratios (RRs) of bleeding reoperation after rapid deployment (RD) versus sutureless (SU) aortic valve replacement (AVR). Pooled analyses from overall patients and from isolated AVR patients are presented. CI, confidence interval; UV, univariate. 


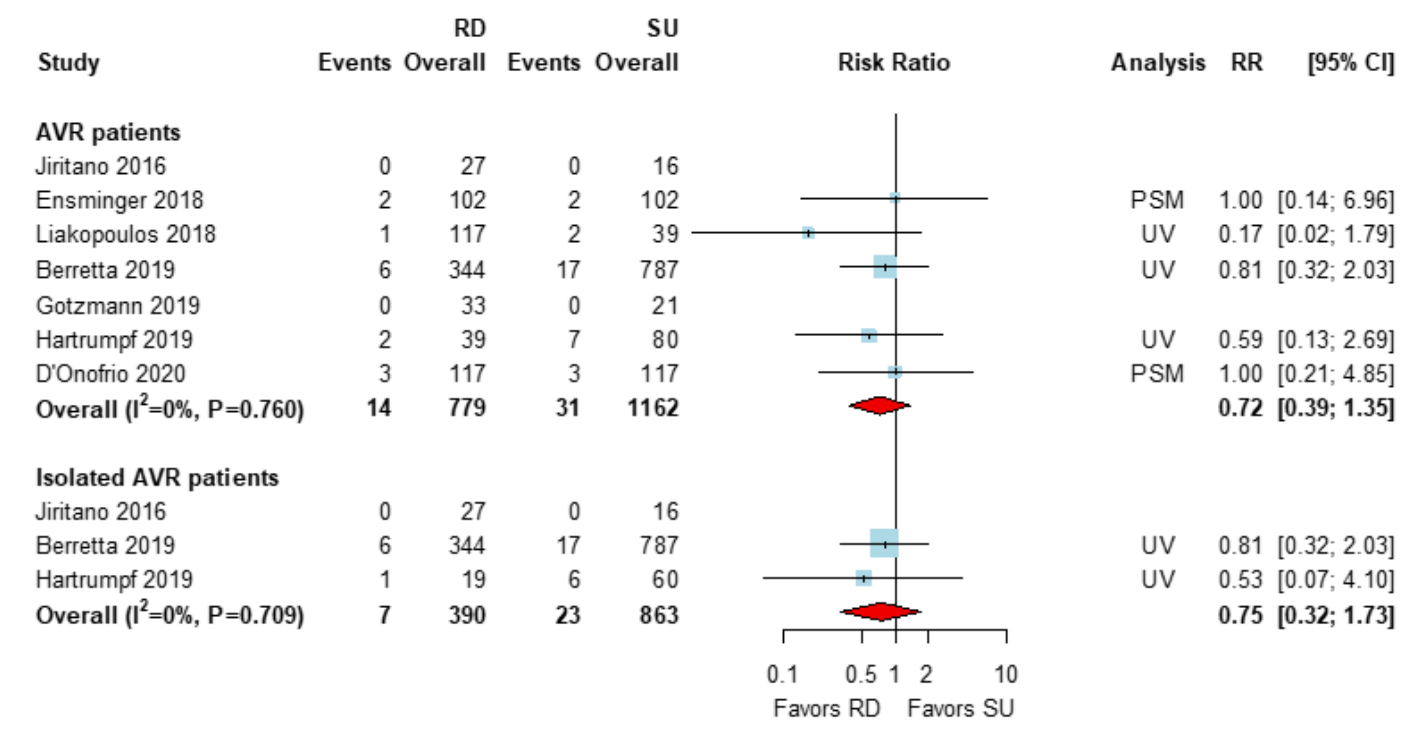

Figure S4 Risk ratios (RRs) of postoperative stroke after rapid deployment (RD) versus sutureless (SU) aortic valve replacement (AVR). Pooled analyses from overall patients and from isolated AVR patients are presented. CI, confidence interval; PSM, propensity score matching; UV, univariate. 

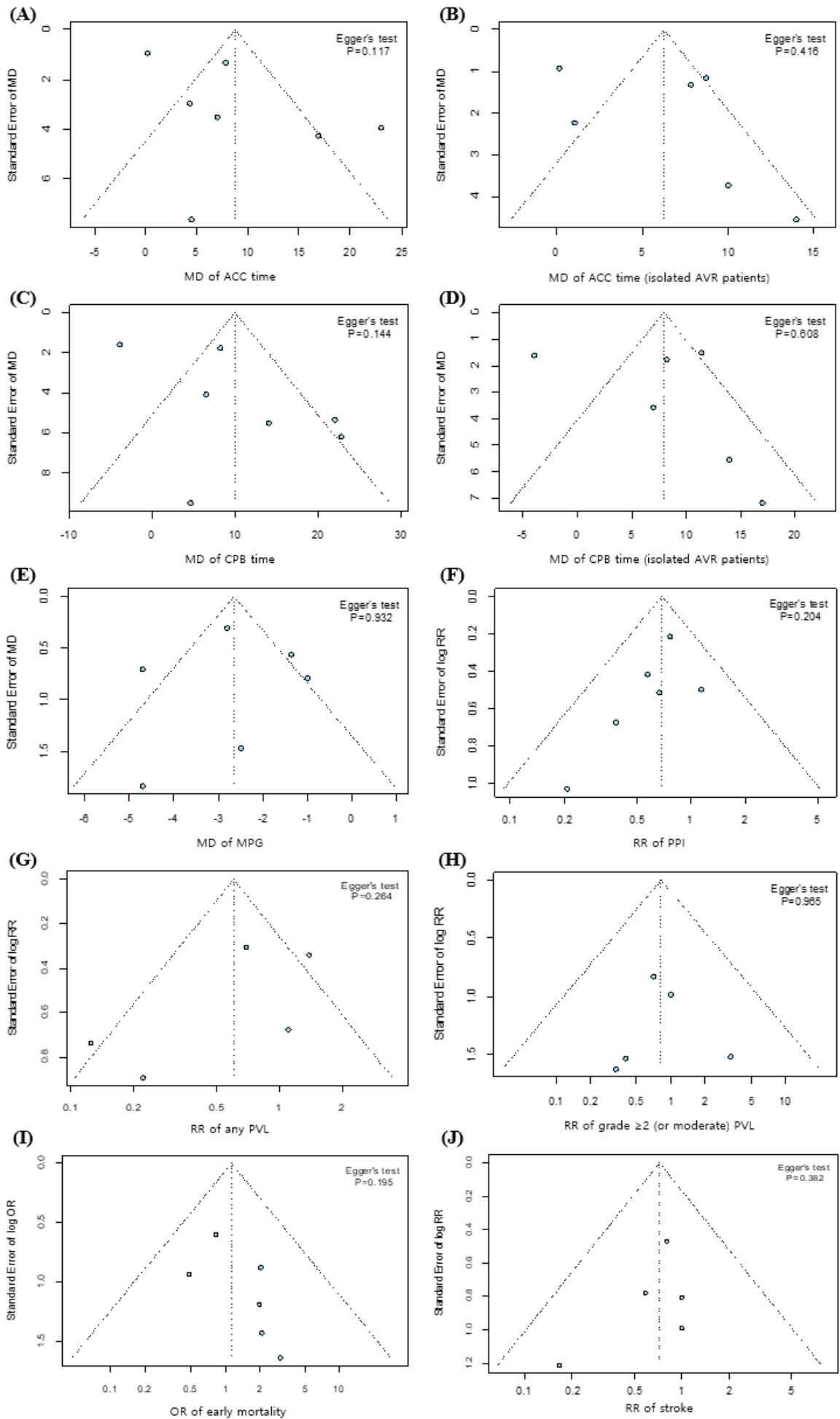

Figure S5 Funnel plots and Egger's test for (A) aortic cross-clamp (ACC) time in overall patients, (B) ACC time in isolated aortic valve replacement (AVR) patients, (C) cardiopulmonary bypass (CPB) time in overall patients, (D) CPB time in isolated AVR patients, (E) mean transvalvular pressure gradient (MPG) of the aortic valve, $(\mathrm{F})$ risk of permanent pacemaker implantation (PPI), (G) risk of any paravalvular leak (PVL), (H) risk of grade $\geq 2$ (or moderate) PVL, (I) early mortality, and (J) stroke. MD, mean difference; RR, risk ratio; OR, odds ratio. 